\title{
Safety of lymphocytes immunotherapy during the COVID-19 outbreak in Wuhan, China
}

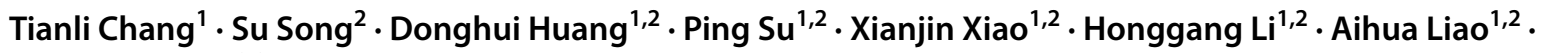 \\ Wenpei Xiang ${ }^{1,2,3}$
}

Received: 22 July 2020 / Accepted: 29 September 2020 / Published online: 2 January 2021

(c) Springer-Verlag GmbH Germany, part of Springer Nature 2021

Keywords SARS-CoV-2 · COVID-19 - Lymphocyte immunotherapy · Recurrent miscarriage

\author{
Abbreviations \\ COVID-19 Coronavirus Disease-2019 \\ SARS-CoV-2 2019 Novel coronavirus \\ RM Recurrent miscarriage \\ LIT Lymphocyte immunotherapy
}

Dear editor,

We read with great interest the informative review article by Cavalcante et al. [1], "Lymphocyte immunotherapy in the treatment of recurrent miscarriage: systematic review and meta-analysis." We pay close attention to the "Safety of immunotherapy with lymphocytes" in the review. Immune dysregulation was an important aspect among multiple etiologies of miscarriage. Lymphocyte immunotherapy was a therapeutic method gained acceptance for clinical application for women suffering from alloimmune recurrent miscarriage. Paternal lymphocyte immunotherapy was attributed to the high expression of anti- paternal cytotoxic antibodies (APCAs), anti-idiotypic antibody (Ab2), mixed lymphocyte reaction blocking antibody (MLR-Bf) and blocking antibody (BAb), these antibodies increased the resistance to the

Tianli Chang and Su Song contributed equally to this work.

Wenpei Xiang

wpxiang2010@gmail.com

1 Institute of Reproductive Health, Tongji Medical College, Huazhong University of Science and Technology, Wuhan, China

2 Center of Reproductive Medicine, Tongji Medical College, Huazhong University of Science and Technology, Wuhan, China

3 Institute of Reproductive Health, Center of Reproductive Medicine, Tongji Medical College, Huazhong University of Science and Technology, Wuhan 430030, China compatibility of human leukocyte antigens (HLA) between father and mother and protected the fetus from toxic effects of the mother's immune system [2,3], suppressed the overactivity of natural killer (NK) cells and T helper-1 (Th-1) pattern of cytokines to induce maternal immune tolerance to fetal allotransplantation in women with alloimmune cause of recurrent pregnancy loss $[4,5]$. The objective of this clinical follow-up is to clarify the security of lymphocytes immunotherapy during the COVID-19 outbreak in Wuhan, China and to provide guidance for subsequent active immunotherapy during the COVID-19 outbreak in the future.

\section{Participants}

To contribute data, our center had to be able to enroll patients treated with allogeneic leukocyte immunization following uniform standards. The inclusion criteria for participants included: (1) the diagnosis was "alloimmune recurrent miscarriage"; (2) alloimmune antibody "blocking antibody" was negative; (3) autoimmune antibody " anti-nuclear antibody, antineutrophil cytoplasmic antibody, rheumatoid factors" were negative; (4) chromosome of couples was normal; and (5) patients were conducted LIT during November1, 2019 to January17, 2020 in Center of Reproductive Medicine, Tongji Medical College, Huazhong University of Science and Technology. Exclusion criteria included: (1) patients with endocrine system diseases were excluded including but not limited to luteal phase deficiency, thyroid disorder, polycystic ovarian syndrome, premature ovarian failure, hyperprolactinemia and diabetes; (2) genital abnormalities such as genital malformation, myoma of uterus, intrauterine adhesion, tubal adhesion, uterine cervical incompetence; and (3) patients with autoimmune disease. 


\section{The study plan}

Through the case management system in the Center of Reproductive Medicine, Tongji Medical College 203 patients were selected as subjects. Clinical 4-month followup were conducted every other week by telephone during February, 2020 to May, 2020. We received 187 complete pieces of feedback (187/203). The follow-up information was demonstrated in Table 1.

\section{Discussion}

Safety of LIT for recurrent miscarriage has not been entirely investigated, although most of the researchers have claimed no side effects on mother and the infants. However, studies regarding the safety of LIT showed that there was a possibility of maternal complications about autoimmunity and the virus susceptibility such as human immunodeficiency viruses, cytomegalovirus and hepatitis virus was increased [1]. Therefore, the risk of infectious disease transmissions was the point of concern. The SARS-CoV-2 infection has become an urgent public health challenge all over the world.
Most of the infected people could recover after 2-3 weeks. However, once infected patients develop severe acute respiratory distress syndrome (ARDS), more than $10 \%$ of patients may deteriorate within a short time and die from multiple organ failure [6]. Recently, a study found that the hyperfunction of CD4 + and CD8 + T cells was associated with the pathogenesis of extremely severe SARS-CoV-2 infection [7]. Th1 and Th2 cells are the main subgroups of fully differentiated CD4 positive T cells. Some noteworthy alterations of T-lymphocyte after LIT, including reduction in the Th1/Th2 ratio increased $\mathrm{CD} 4+/ \mathrm{CD} 8+$ ratio and decreased $\mathrm{CD} 8+\mathrm{T}$ cell percentage, made a combined effect on regulation of maternal tolerance and prevention of fetus rejection, which could consequently increase the pregnancy success [8, 9]. However, whether these changes in the immune system were predisposing factors to SAR-COV-2 infection was not clear. The demographic characteristics in Table 1 indicated that all the patients were not suffered from COVID-19 during the COVID-19 outbreak in Wuhan while Wuhan's overall infection rate is $0.55 \%(50,340 / 9,080,000$, data cutoff on June 24, 2020). It seemed that lymphocyte immunotherapy was safety for all the patients who had a residential history of Wuhan or potential COVID-19 exposure in the early stage of epidemic of Wuhan, while patients' awareness of protection might

Table 1 Demographic characteristics of study participants $(n=187)$

\begin{tabular}{|c|c|}
\hline Characteristics & Lymphocyte immunotherapy (LIT) \\
\hline Residence & Wuhan \\
\hline Age, (years) (mean $\pm \mathrm{SD})$ & $31.35 \pm 0.3418$ \\
\hline Blocking antibody positive conversion*, $n(\%)$ & $124(66.31 \%)$ \\
\hline Pregnancy, $n(\%)$ & $70(37.4 \%)$ \\
\hline Type of Lymphocyte immunotherapy (LIT) & Paternal LIT \\
\hline Therapeutic schemes & $\begin{array}{l}2-8 \text { times before the planned preg- } \\
\text { nancy with } 2 \text { weeks of intervals }\end{array}$ \\
\hline \multicolumn{2}{|l|}{ Time of infusion within 10 weeks*, $n(\%)$} \\
\hline$\leq 4$ times with 2 weeks of intervals & $89(47.5 \%)$ \\
\hline 4 times with 2 weeks of intervals & $96(51.4 \%)$ \\
\hline$\geq 4$ times with 2 weeks of intervals & $2(1.1 \%)$ \\
\hline Dose of lymphocyte per treatment & $2-5 \times 10^{7}$ lymphocyte/mL (NS) \\
\hline Injection site & Intradermal injection of the forearms \\
\hline \multicolumn{2}{|l|}{ Immunosuppressive medication history, $n(\%)$} \\
\hline Prednisone & $18(9.6 \%)$ \\
\hline Dexamethasone & $3(1.6 \%)$ \\
\hline Cyclosporine & $2(1.1 \%)$ \\
\hline \multicolumn{2}{|l|}{ Complications } \\
\hline $\begin{array}{l}\text { Autoimmune disorders (Hyperthyroidism, Hepatitis, Purpura, Systemic lupus erythematosus, Rheuma- } \\
\text { toid arthritis, Chronic urticaria), } n(\%)\end{array}$ & $1 * *$ \\
\hline Alloimmune disorders (Viral Transmission, Flu-like symptoms, Transfusion reaction), $n$ (\%) & 0 \\
\hline Diagnosis of COVID-19, $n(\%)$ & 0 \\
\hline
\end{tabular}

*The antibody detection and treatment were discontinued due to city lockdown

** One patient suffered from chronic urticaria when she was at 11 gestational weeks. Oral low-dose cyclosporine and antiallergic drugs were applied for 2 weeks 
not be sufficient at that time. In consideration of factors such as insufficient sample size, effectiveness of protective measures and inconsistency of exposure risk, we need longterm follow-up, multicenter trials with larger sample size, case-control or prospective studies to confirm the security and risk of lymphocyte immunotherapy.

Acknowledgements Thanks for all of the staffs who participated in the follow-up work. Especially, we want to express our deep respect to all first-line healthcare workers for their dedication in the fight against SARS-CoV-2.

Author contributions WX designed the plan of the follow-up. TC, SS, DH, PS and XX performed the study plan, TC and SS analyzed the data and co-authored the manuscript. HL and AL provided their invaluable contributions in critically revising the manuscript and providing guidance for important intellectual content.

Funding This work was supported by HUST COVID-19 Rapid Response Call Program (2020kfyXGYJ056) and Hubei Provincial Emergency Science and Technology Program for COVID-19 (2020FCA037).

\section{Compliance with ethical standards}

Conflict of interest The authors declare that they have no competing financial interests or personal relationships that could have appeared to influence the work reported in this paper.

\section{References}

1. Cavalcante MB, Sarno M, Araujo Junior E, Da Silva CF, Barini $\mathrm{R}$ (2017) Lymphocyte immunotherapy in the treatment of recurrent miscarriage: systematic review and meta-analysis. Arch Gynecol Obstet 295(2):511-518. https://doi.org/10.1007/s0040 4-016-4270-z

2. Pandey MK, Thakur S, Agrawal S (2004) Lymphocyte immunotherapy and its probable mechanism in the maintenance of pregnancy in women with recurrent spontaneous abortion. Arch Gynecol Obstet 269(3):161-172. https://doi.org/10.1007/s0040 4-003-0560-3

3. Takakuwa K, Kanazawa K, Takeuchi S (1986) Production of blocking antibodies by vaccination with husband's lymphocytes in unexplained recurrent aborters: the role in successful pregnancy. Am J Reprod Immunol Microbiol 10(1):1-9. https://doi. org/10.1111/j.1600-0897.1986.tb00001.x

4. Zhao X, Jiang Y, Wang L, Li Z, Li Q, Feng X (2018) Advances in Understanding the Immune Imbalance between T-Lymphocyte Subsets and NK Cells in Recurrent Spontaneous Abortion. Geburtshilfe Frauenheilkd 78(7):677-683. https://doi. org/10.1055/a-0634-1813

5. Liu M, Zhen X, Song H, Chen J, Sun X, Li X et al (2017) Lowdose lymphocyte immunotherapy rebalances the peripheral blood Th1/Th2/Treg paradigm in patients with unexplained recurrent miscarriage. Reprod Biol Endocrinol: RB\&E 15(1):95. https:// doi.org/10.1186/s12958-017-0315-9

6. Chen N, Zhou M, Dong X, Qu J, Gong F, Han Y et al (2020) Epidemiological and clinical characteristics of 99 cases of 2019 novel coronavirus pneumonia in Wuhan, China: a descriptive study. Lancet 395(10223):507-513. https://doi.org/10.1016/s0140 $-6736(20) 30211-7$

7. Wang F, Hou H, Luo Y, Tang G, Wu S, Huang M et al (2020) The laboratory tests and host immunity of COVID-19 patients with different severity of illness. JCI Insight. https://doi.org/10.1172/ jci.insight. 137799

8. Liang P, Mo M, Li GG, Yin B, Cai J, Wu T et al (2012) Comprehensive analysis of peripheral blood lymphocytes in 76 women with recurrent miscarriage before and after lymphocyte immunotherapy. Am J Reprod Immunol 68(2):164-174. https://doi.org/1 0.1111/j.1600-0897.2012.01141.x

9. Wu L, Luo LH, Zhang YX, Li Q, Xu B, Zhou GX et al (2014) Alteration of Th17 and Treg cells in patients with unexplained recurrent spontaneous abortion before and after lymphocyte immunization therapy. Reprod Biol Endocrinol: RB\&E 12:74. https://doi.org/10.1186/1477-7827-12-74

Publisher's Note Springer Nature remains neutral with regard to jurisdictional claims in published maps and institutional affiliations. 